\title{
Infrapatellar Ganglion Cyst of the Knee Fat Pad in a Child with Juvenile Idiopathic Arthritis
}

MASAKI SHIMIZU, MD, PhD, Department of Pediatrics; JUNSUKE NAKASE, MD, PhD, Department of Orthopedic Surgery; AKIHIRO YACHIE, MD, PhD, Department of Pediatrics, School of Medicine, Institute of Medical, Pharmaceutical, and Health Sciences, Kanazawa University, Kanazawa, Japan. Address correspondence to Dr. M. Shimizu, Department of Pediatrics, School of Medicine, Institute of Medical, Pharmaceutical, and Health Sciences, Kanazawa University, 13-1 Takaramachi, Kanazawa 920-8641, Japan. E-mail: shimizum@staff.kanazawa-u.ac.jp. This is a case report, therefore it was unnecessary to receive ethics board approval. The patient gave written informed consent to publish the material. J Rheumatol 2019;46:112; doi:10.3899/jrheum.180406

Ganglion cysts of the knee are rare, but they can appear in cases of juvenile idiopathic arthritis (JIA). Magnetic resonance imaging (MRI) identifies them when there is persistent pain without swelling.

A 10-year-old girl with JIA diagnosed at 2 years of age presented with arthralgia and swelling of the right knee. She had been disease-free for 3 years, and JIA relapse was diagnosed. Methotrexate (MTX) was readministered, but arthritis of the right elbow and right third and fifth proximal interphalangeal joint appeared. Adalimumab was added to MTX and her symptoms, other than mild right knee pain, resolved. Right knee MRI revealed a multilobular cystic lesion with a thin membrane, which was apparent between the infrapatellar fat pad and anterior cruciate ligament (Figure 1). Arthroscopic examination revealed that the mass arose from the infrapatellar fat pad. Complete cystic mass excision was performed. Histological diagnosis was consistent with a ganglion cyst. After cystic mass excision, her right knee pain resolved.

Ganglion cysts are benign cystic tumor-like lesions surrounded by dense connective tissue capsules containing myxoid matrix ${ }^{1,2}$. Ganglion cysts rarely arise from the infrapatellar fat pad ${ }^{1,2,3,4}$. Clinical symptoms of ganglion cysts, including knee pain, decreased range of motion, clicking or popping sensations and a palpable mass are nonspecific and depend on their size and location ${ }^{1,2,3,4}$. These symptoms overlap with and mimic the common complaints of patients with JIA. MRI of the knee should be considered in patients with JIA with symptoms of persistent pain without swelling, with clicking, popping, or the presence of a mass. Although ganglion cysts of the knee are rare, they can be identified by MRI as was seen in this patient.

\section{REFERENCES}

1. Nikolopoulos I, Krinas G, Kipriadis D, Ilias A, Giannakopoulos A, Kalos S. Large infrapatellar ganglionic cyst of the knee fat pad: a case report and review of the literature. J Med Case Rep 2011;5:351.

2. Takahashi T, Kimura M, Ohsawa T, Yamaguchi N, Takeshita K. A case of infrapatellar fat pad ganglion of the knee. Open Orthop J 2017;11:1142-6.

3. Sugiura K, Suzue N, Matsuura T, Hamada D, Goto T, Takata Y, et al. Ganglion cyst arising from the infrapatellar fat pad in a child. J Med Invest 2015;62:245-7.

4. Bisicchia S, Savarese E. Infra-patellar fat pad cysts: a case report and review of the literature. Muscles Ligaments Tendons J $2013 ; 2: 305-8$
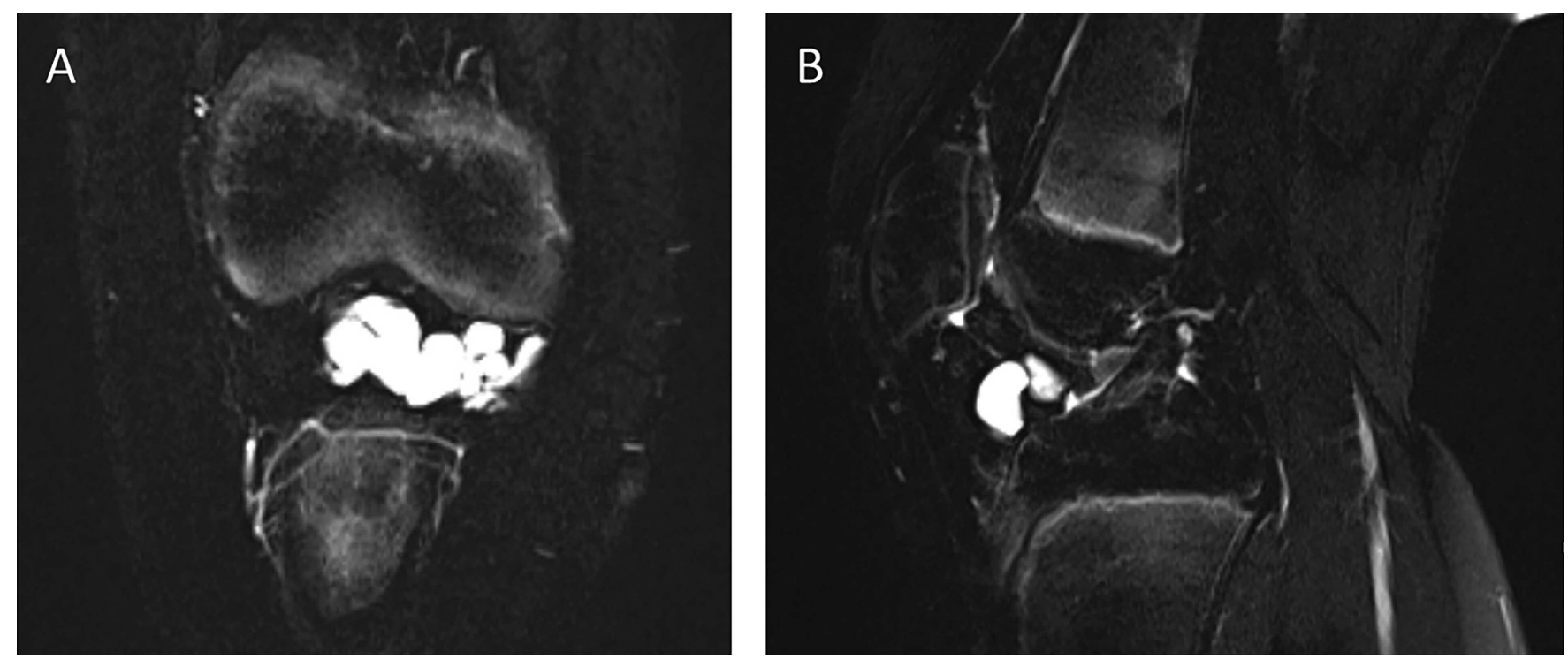

Figure 1. Magnetic resonance imaging findings (fat-suppressed, T2-weighted images). The well-circumscribed high-intensity multilobular cystic lesion is apparent between the infrapatellar fat pad and the anterior cruciate ligament. A. Coronal view. B. Sagittal view.

Personal non-commercial use only. The Journal of Rheumatology Copyright $\odot$ 2019. All rights reserved 